\title{
Le dispositif cinématographique chez Claude
} Simon

Bérénice Bonhomme

\section{OpenEdition}

1 Journals

Édition électronique

URL : http://journals.openedition.org/ccs/639

DOI : $10.4000 /$ ccs. 639

ISSN : 2558-782X

Éditeur :

Presses universitaires de Rennes, Association des lecteurs de Claude Simon

\section{Édition imprimée}

Date de publication : 31 décembre 2009

Pagination : 85-98

ISBN : 9782354120580

ISSN : $1774-9425$

\section{Référence électronique}

Bérénice Bonhomme, "Le dispositif cinématographique chez Claude Simon », Cahiers Claude Simon [En ligne], 5 | 2009, mis en ligne le 21 septembre 2017, consulté le 20 avril 2019. URL : http:// journals.openedition.org/ccs/639; DOI : 10.4000/ccs.639 


\title{
Le dispositif cinématographique chez Claude Simon
}

\author{
Bérénice BONHOMME
}

Dans l'œuvre simonienne, on peut relever de multiples références au monde cinématographique, que ce soit par simples allusions ou de façon plus marquée par l'emploi d'un vocabulaire spécifique et technique. Dans chacun des romans, en tout cas, le cinéma fait son apparition d'une manière ou d'une autre, au détour d'une phrase comme dans La Route des Flandres ou bien au cours d'un développement plus long comme dans Les Géorgiques ou Le Tramway. Comment, en outre, ne pas être frappé par le découpage cinématographique qui clôt Le Jardin des Plantes et qui souligne de façon presque pédagogique, quoique légèrement distanciée, la place du cinéma dans l'œuvre ? Claude Simon déclare dans un questionnaire des Cahiers du cinéma:

Ce que l'on peut en dire c'est que pour moi, comme pour tous, le cinéma a enrichi la vision que nous avons des choses (angles et distances des " prises de vues", panoramiques, plans fixes, travellings, gros plans). Et naturellement cette nouvelle façon de voir se retrouve dans ce que j'écris. ${ }^{2}$

Auteur de "Triptyque" de Claude Simon. Du livre aufilm. Une esthétique du passage (Fasano/Paris, Schena Editore/Presses de l'Université de Paris-Sorbonne, 2005) et de Claude Simon, l'écriture cinématographique (L'Harmattan, 2005). A soutenu en 2008 une thèse de doctorat sur Claude Simon, la passion du cinéma (voir rubrique « Actualité de l'œuvre »).

2 «Questions aux romanciers », Les Cahiers du cinéma, décembre 1966, p. 103. 
L'auteur souligne lui-même l'empreinte qu'ont laissée les caractéristiques des images cinématographiques (cadre, angle, mouvement de caméra) dans son écriture. Remarquons également avec quelle insistance Claude Simon rappelle l'aspect technique du cinéma (panoramique, travelling). La relation qui existe entre le spectateur et l'image apparaît, enfin, fondatrice. L'auteur se présente comme un spectateur de cinéma et met au cœur de cette expérience la vision. Nous étudierons donc ici la place du dispositif cinématographique dans l'œuvre.

\section{Vocabulaire technique}

Même si Claude Simon se présente comme un spectateur de cinéma comme les autres, lorsqu'on lit les romans, on se rend vite compte que ses connaissances en ce qui concerne les techniques cinématographiques dépassent largement la moyenne. On peut remarquer dans l'œuvre un véritable vocabulaire technique du cinéma, concernant à la fois la phase de tournage et la projection. Ainsi les métiers des techniciens de cinéma sont très bien représentés, par exemple " Metteur en scène » $(T, 791,822,828 ; G, 385)$, " Machinistes » $(T, 791,822,850)$, «Électriciens » $(T, 791)$, «Accessoiriste » $(V, 164 ; J P, 1178)$, «Vedette de cinéma » $(R F, 290 ; P, 41 ; B P, 662$; $C C, 155)$ et "Opérateur» $(P, 422 ; T, 805)$. De même, l'évocation du matériel constitue un champ lexical à part entière. On remarque "Caméra " (très fréquent), "Studio " $(T, 821,822)$, "Celluloïd " (Herbe, $134 ; R F, 290)$, ou encore "Appareil de projection» ( $T$, $759,804,864 ; G, 206)$. Enfin les techniques cinématographiques sont mentionnées, comme "Plan fixe» $(J P, 1173,1174)$, «Angles de prise de vues " $(C R, 66)$, "Truquage de cinéma " $(R F, 241)$ et les images « mal tirées et surexposées » $(R F, 239)$.

Ce relevé, bien que très parcellaire, permet de rendre compte de la richesse du vocabulaire cinématographique utilisé par Claude Simon. Bien sûr, on constate une surreprésentation de ce champ lexical dans le roman Triptyque qui traite plus spécifiquement du cinéma et dans le découpage qui clôt Le Jardin des plantes, mais c'est une isotopie qui traverse toute l'œuvre, depuis $L a$ Corde raide jusqu'au Tramway. On aurait pu s'attendre à ce que le vocabulaire technique gagne en importance et en précision après le tournage de 
1975 (le court-métrage L'Impasse inspiré de Triptyque ${ }^{3}$ ), qui a été la première - et à ma connaissance la seule - expérience de tournage pour Claude Simon, mais cela reste assez uniforme (les romans précédant cette date faisant déjà une large place à ce champ lexical), ce qui montre combien l'intérêt pour le cinéma est une des constantes fortes de l'œuvre.

Tout d'abord, on ne peut qu'être frappé par la précision technique de certains des termes employés par Claude Simon et les savoirs qu'ils présupposent. L'auteur s'appuie fréquemment sur des connaissances qui lui viennent de sa pratique de la photographie. Ainsi, il sait que le support de la pellicule est constitué de celluloïd et il qualifie donc à deux reprises les actrices de " créatures de celluloïd» (Herbe, $134 ; R F, 290)$. Il utilise également des termes appartenant uniquement au champ du cinéma comme " travelling " $(T$, $834 ; J P, 1169)$, « zoom » (JP, 1169), « visa d'exploitation» $(I, 78)$ et bien d'autres dans un contexte approprié, ce qui implique plus qu'un vernis de connaissances. Certes, quelques imprécisions demeurent, comme l'utilisation dans Triptyque du terme « cinéastes » au pluriel pour désigner l'ensemble des techniciens massés derrière l'appareil de prise de vue, alors que "cinéaste » est couramment utilisé pour désigner le réalisateur d'un film $(T, 761)$. De même, dans Le Palace, c'est le « régisseur» $(P, 422)$ qui donne le signal de l'entrée dans le champ alors que traditionnellement c'est plutôt le travail du metteur en scène (ou en tout cas d'un de ses assistants). Mais ce ne sont que de très légères inexactitudes et l'accumulation des détails dénote assez l'intérêt de l'auteur pour le " comment ça marche » du cinéma.

Cependant, cet intérêt semble ambigu. L'auteur paraît éprouver un véritable plaisir à décrire la machine cinéma, mais on ne peut qu'être frappé par la phrase lapidaire de La Corde raide : "Il n'y a qu'à voir ce qui se passe avec le cinéma. Les gens qui font du cinéma deviennent maintenant de plus en plus forts en technique (bonne photographie, angles de prises de vue, effets spéciaux) et de plus en plus nuls en cinéma. " $(C R, 66)$. De fait, les films décrits dans Triptyque ne sont manifestement pas des chefs-d'œuvre. L'insistance sur le dispositif technique vide de son sens le récit filmique. Il n'en reste

\footnotetext{
${ }^{3}$ Voir Claude Simon, Euvres, p. 1240-1245, Bérénice Bonhomme, "Triptyque » de Claude Simon. Du livre au film. Une esthétique du passage, op. cit., Mireille Calle-Gruber (éd.), Les Triptyques de Claude Simon ou l'art du montage, Presses Sorbonne Nouvelle, 2008.
} 
pas moins que lorsque Claude Simon tentera pour la première fois une adaptation d'un de ses livres \{La Route des Flandres), il proposera un cinéma très technique, utilisant un large éventail des possibilités matérielles de l'époque, sans pour autant évacuer le sens.

\section{Tournage et projection}

Claude Simon privilégie clairement deux temps dans le processus de fabrication et de vie du film, le tournage et la projection.

\section{Le tournage}

Lorsqu'une image cinématographique est décrite, Claude Simon revient volontiers sur le moment même de sa création, ce moment où elle s'inscrit sur la pellicule. Une image porte toujours en elle le présent du tournage, l'instant de son inscription effective, toujours prêt à affleurer :

Dans son mouvement tournant, la glace a reflété pendant une fraction de seconde la pénombre du studio où dans un camaïeu brun est apparue la forme noire de la caméra de prises de vue aux yeux multiples, ses tambours, son socle, ses câbles, et les visages attentifs quoique imprécis des techniciens de l'équipe massés derrière elle. $(T, 821)$

Dans L'Acacia, les images des hommes partant à la guerre portent en creux celle de "l'opérateur» $(A, 133)$ que l'on fait surgir derrière sa caméra. C'est encore plus évident dans Triptyque qui rend plus particulièrement compte de la thématique cinématographique. Prenons un exemple :

Le plan suivant a dû faire l'objet de tous les soins du metteur en scène car il suppose une série de mouvements compliqués de la caméra s'approchant, s'éloignant, se rapprochant de nouveau, cadrant successivement : une serviette de cuir à fermoir métallique, puis les deux protagonistes assis sur une banquette, comme celle d'un bar, puis l'un ou l'autre de leurs visages et, à la fin, de nouveau la serviette, mais ouverte cette fois, et dont on peut voir l'intérieur rempli de liasses de billets. $(T, 828)$ 
Nous sommes face à un plan d'images déjà tournées. Et pourtant, ce qui est mis en évidence ce sont les mouvements de la caméra au moment du tournage qui rendent possible l'existence de telles images.

Le moment du tournage est décrit avec un certain réalisme, ce qui d'ailleurs ne laisse pas de surprendre. En analysant des extraits de Triptyque, on décèle déjà une certaine familiarité avec ce temps de processus de fabrication d'un film :

[...] l'homme de nouveau lui aussi dans sa posture familière, une main sur ses reins, [...], la voix au fort accent anglais disant Maintenant vous devriez essayer de dormir voulez-vous que je vous donne ces cachets ?, la voix du metteur en scene criant alors Coupez, et les lumières des projecteurs s'éteignant les unes après les autres sur les passerelles invisibles dans les ténèbres des cintres qui se referment peu à peu. Pendant un moment on peut entendre le remue-ménage des appareils que l'on change de place, la voix du metteur en scène qui commente laprise de vues ou donne ses ordres, et les discussions des techniciens qui s'affairentpour les exécuter. L'acteur anglais s'est assis en arrière de la caméra et à l'écart, dans un fauteuil pliant. Renvoyant d'un mot la maquilleuse qui s'est approchée, il reste là [...]. Sur le sol serpentent et s'embrouillent une multitude de câbles dans leurs gaines de caoutchouc noir. L'actrice est restée étendue sur le lit dans la même position, et la maquilleuse qu'a renvoyée son partenaire se penche sur son visage où elle promène délicatement les soies d'un pinceau. L'un des machinistes qui s'apprêtait à vider dans un sac le contenu du cendrier posé sur la petite table à la tête du lit suspend son geste sur un ordre et s'applique à répartir de nouveau sur la coupe de porcelaine les trois cigarettes écrasées et tordues à l'extrémité ensanglantée. Lorsque les rampes des projecteurs se rallument l'une après l'autre, l'appareil de prise de vue a été roulé en avant et dans le viseur s'encadrent en gros plan la tête, le buste et les bras de la femme qui feuillette en arrière [...] le livre dont, un peu plus tôt, elle a interrompu la lecture ${ }^{4}$. $(T, 822-823)$

Claude Simon rend compte du moment où l'on finit de tourner un plan et où l'on passe au suivant. C'est donc un temps de mise en place. Beaucoup de détails sonnent juste. Ainsi, c'est le metteur en scène qui clôt la prise avec son lapidaire : " coupez ». L'aspect très organisé, voire un peu militaire, que peut avoir un tournage,

\footnotetext{
${ }^{4}$ C'est moi qui souligne.
} 
est bien rendu. Les ordres sont donnés et les techniciens s'affairent pour les exécuter. Claude Simon insiste aussi sur l'importance de la continuité des accessoires (les trois cigarettes) pour éviter des erreurs de raccord. Profitant de la mise en place technique, la maquilleuse procède à des raccords maquillage, puis le tournage reprend. Le studio est bien décrit avec ses " rampes de projecteurs " et la multitude de « câbles » qui courent sur le sol. Enfin, l'auteur relate l'ambiance particulière d'un changement de plan, lorsque l'équipe technique passe du silence complet - massée derrière la caméra, les " visages attentifs " (821) au jeu des acteurs - à une activité frénétique, prise de possession du plateau et « remue ménage des appareils que l'on change de place $»$. Cependant, certains détails semblent légèrement s'éloigner de la réalité d'un tournage. Ainsi, les rôles du réalisateur et de son premier assistant sont confondus - normalement c'est le premier assistant qui est chargé de donner les directives aux techniciens -, ce qui peut sans doute s'expliquer par l'intérêt que Claude Simon portait à la technique, n'imaginant pas réaliser un film sans en diriger les techniciens.

Dans ces descriptions sur la façon dont se déroule un tournage, un élément est davantage mis en exergue : le studio. On peut y voir un hommage au cinéma classique hollywoodien qui a bercé la jeunesse de l'auteur. En outre, il n'est pas étonnant de retrouver ici un des éléments les plus frappants du cinéma, permettant de jouer à l'infini sur la perception de l'espace et de ce que l'on offre à voir au spectateur. Le studio se fonde ainsi sur un fantasme d'omnipotence de la vision. C'est un dispositif qui permet de tout voir et de tout montrer, de multiplier les angles de vue, même les plus inimaginables (Claude Simon suggérant dans son découpage de La Route des Flandres de mettre la caméra à la place du mur). Cette tension du regard est à associer aux multiples évocations de dessins de décors en coupe, nous permettant ainsi de voir ce qu'ils contiennent :

Une coupe longitudinale pratiquée derrière l'un ou l'autre des murs dans la suite des chambres qui se succèdent à droite et à gauche permettrait de voir les pièces toutes semblables, aux mobiliers identiques, les unes vides, les autres occupées par des hommes ou des femmes, certains en train de se laver les mains ou de se brosser les dents, d'autres défaisant ou refermant des valises, d'autres écrivant des lettres, d'autres encore assis dans les fauteuils ou étendus dé- 
chaussés sur les lits en train de feuilleter des journaux ou des magazines. (CC, 222-223)

La construction d'un espace factice qui est là pour s'exhiber fait évidemment penser aux studios. On sait que le cinéma a utilisé ce type de décors afin de rendre possible des plans compliqués en mouvement, donnant à voir tous les occupants d'une maison (comme dans le film de Jerry Lewis Le Tombeur de ces dames). À l'opposé, Claude Simon évoque également des façades de maisons, sans rien derrière, dont le cinéma fait également grande consommation, « suite de façades restées debout, sans rien derrière, comme un décor de théâtre aux fenêtres vides à travers lesquelles on peut voir le ciel " $(J P, 1019)$ et qui rappellent les décors hollywoodiens.

Si la thématique du studio est ainsi récurrente, c'est en grande partie à cause des questions qu'elle pose sur la réalité et le factice : «Dans le visage renversé en arrière les yeux sont ouverts, regardant fixement le plafond de la chambre ou plutôt les cintres du studio de prise de vues avec leurs câbles, leurs treuils, leurs passerelles garnies de projecteurs. » $(T, 790)$. À la vision attendue, suggérée par l'image (on suppose le plafond de la chambre) se substitue celle des outils de l'illusion. Tout est contaminé par le soupçon de la facticité :

La vue légèrement plongeante est prise d'un point situé à un mètre environ en arrière de la tête du lit, ce qui donne à penser soit que le lit a été tiré au milieu de la pièce, soit, comme il est plus probable, que le lit se trouve au centre de l'espace vide du plateau du studio fermé à l'extrémité opposée par un décor composé de panneaux, également nus, au centre desquels s'ouvre le rectangle d'une fenêtre où ne s'encadre rien d'autre qu'un ciel sans nuages, d'une teinte uniforme comme passée par un peintre en bâtiment sur une toile située à quelques mètres en arrière de la fenêtre. ( T, 790)

Ainsi tout n'est que manipulation et illusion. Seules deux explications viennent à l'esprit : soit l'on a triché sur la disposition des meubles de la chambre, soit il n'existe même pas de chambre mais uniquement cet espace vide du studio. Soulignons l'importance de cette fenêtre qui donne sur un ciel factice, peint sur un des panneaux du décor. L'évocation d'un ciel peint revient souvent dans l'œuvre de Claude Simon, par exemple dans La Route des Flandres où est 
décrit « l'immuable archipel des nuages suspendus, ou peut-être tout simplement peints, dans le ciel $»(R F, 316)$. Ainsi le studio pourrait symboliser le mensonge de l'univers et la déréliction de l'homme prisonnier d'un espace fragmenté. Les décors au cinéma, en effet, ne sont pas construits pour durer, décors de passage où il est impossible de se fixer, ce qui accentue le malaise propre à la condition humaine :

[...] il se dégage de l'ensemble une sensation de vacuité, d'anonymat et de désolation, comme si les protagonistes n'étaient là que de passage, dans un cadre provisoire et factice auquel ils n'ont aucune part, disposé la veille par des machinistes prêts à le démonter et isolé par les projecteurs comme un minuscule et éphémère îlot de lumière dans l'immensité du cosmos ou, plus simplement, d'un vaste hangar de studio, tout aussi noir et tout aussi vide. $(T, 850)$

\section{Laprojection}

Claude Simon fait aussi preuve d'une grande familiarité avec la technique mise en oeuvre pour projeter un film. Il évoque tout le matériel nécessaire à une projection cinématographique : " projecteur» $(B P ; 24)$ installé dans la " cabine de projection » $(T, 772)$, " pellicule cinématographique » (789) et " toile de l'écran » $(C C$, 102) protégée par un "rideau " $(G, 206)$ que l'on soulève à l'aide d'une " manivelle» et de "poulies» (207). Claude Simon appartient à l'épistémé de la civilisation mécanique du début du vingtième siècle. On peut d'ailleurs signaler dans presque tous ses romans la présence d'un train ou de son substitut le tramway. Ce contexte de modernité explique en partie la fascination que l'on note dans toute l'œuvre pour le dispositif cinématographique et en particulier l'appareil de projection, qui est décrit comme une petite machine caractérisée par ses pannes et ses bruits de "grésillements" $(T, 775$. $G$, $206)$ et de "crépitements" $(G, 212)$ (une vraie petite locomotive en somme). Cette insistance sur le bruit de l'appareil de projection nous renvoie à sa matérialité, car on ne peut jamais vraiment oublier sa présence. Concret, il est décrit de la façon la plus réaliste possible, Claude Simon évoquant le " mécanisme de l'appareil " $(T, 804)$ et son " moteur" ou s'interrogeant sur le défilement et la " fréquence encore imparfaite des images" $\left(H_{e r b e}{ }^{5}, 52\right)$ propre à la projection

\footnotetext{
${ }^{5}$ Collection « Double».
} 
des films du début du cinéma. En effet, c'est par ces imperfections même et ces pannes que le projecteur prend tout son intérêt, et l'auteur semble bien les connaître, en tout cas au moins les plus courantes. Il évoque ainsi le défaut de fixité qui provoque un "flou sautillant " $\{G, 212)$ sur les images, les problèmes sonores comme la "partie sonore mal synchronisée avec le déroulement des images" (Herbe, 71) et les problèmes de défilement, avec les saccades de la pellicule qui finit par se coincer et prendre feu dans Triptyque. Il montre aussi les attaques du temps contre la pellicule qui apparaît comme " éraillée " \{Herbe, 135). Il est intéressant de constater que ces défectuosités sont encore aujourd'hui celles qu'on demande à l'apprenti projectionniste de signaler et de réparer dans la mesure du possible lors du passage du CAP.

La vie d'opérateur dans l'œuvre simonienne n'est donc pas de tout repos. Il faut dire que Claude Simon privilégie des situations de projections difficiles, dans des cinémas populaires ou même des cinémas ambulants. Loin d'être une technique sans faille qui permettrait que chaque séance ressemble à la suivante, la projection est ramenée à un statut artisanal. Chaque projection transforme le film selon les multiples incidents et les choix du projectionniste, l'image se zébrant progressivement « de rapides griffures " $\{A, 314)$, le rythme évoluant selon les préférences de l'opérateur qui peut "pass[er] au ralenti » $\{$ Tram, 65) ou "projet[er] à l'accéléré " $\{G, 385)$ ou au moment de raccorder faire sauter " un certain nombre d'images" $(T, 865)$. On voit à quel point un film peut évoluer, sans même parler des multiples pannes qui jalonnent et interrompent sans arrêt les projections. Si l'on peut parler de présent de l'écriture pour le tournage, la projection est un présent de la lecture et fait du lecteur un partenaire actif. De fait, tout comme le projectionniste, le lecteur peut influer sur le rythme du livre (en faisant varier sa vitesse de lecture ou en sautant des pages), au contraire du spectateur de cinéma sous les yeux duquel se déroule un film dans un temps dont il n'est pas le maître. C'est pourquoi, je propose de voir, dans le cadre du dispositif cinématographique, un double du lecteur non seulement dans le spectateur mais aussi dans l'opérateur projectionniste. Mais le lecteur (de même que le projectionniste et que le spectateur) est également influencé par les circonstances de sa lecture et les événements extérieurs qui la gênent et l'interrompent. Claude Simon déclare, 
expliquant ce qu'il entend par le présent de l'écriture : " Je ne l'ai clairement compris que lorsque j'écrivais La Bataille de Pharsale: un jour dans l'appartement situé au-dessus du mien mon voisin faisait marcher un électrophone qui me gênait, alors j'ai tout simplement intégré cet "événement" dans mon texte. $»^{6}$. Ces événements et accidents, la projection n'en manque pas et ils finissent par devenir la trame même du film qui est donné à voir. La projection peut donc être comprise comme un moment de création, mimant le pouvoir de réécriture que Claude Simon reconnaît à ses lecteurs.

Enfin, notons que, dans le dispositifde la projection de film, un élément semble avoir une importance plus grande. En effet, l'auteur met l'accent sur le faisceau de lumière qui sort de la cabine de projection, "pinceau blafard » et " lumineux» $(T, 760)$ qui, passant audessus de la tête des spectateurs, transporte l'image jusqu'à l'écran :

Des poussières aussi et des volutes bleuâtres de fumée qui s'élèvent en se déroulant et se renroulant sur elles-mêmes sont suspendues dans le faisceau de lumière qui jaillit de la cabine de projection et va de celle-ci à l'écran par-dessus les têtes des spectateurs. Des striures mouvantes dont l'intensité va du blanc au noir balaient le pinceau lumineux en fonction des déplacements des ombres et des lumières sur l'écran. $(T, 760)$

Le chemin lumineux du projecteur à l'écran est mis en évidence, bien visible dans les poussières et volutes de fumée. Notons aussi l'insistance avec laquelle sont soulignés les liens étroits qui existent entre ce pinceau et l'image présente sur l'écran à travers un même rythme lumineux.

Jacques Aumont distingue deux sortes d'images ${ }^{7}$, les « images opaques » qui résultent d'un dépôt comme la peinture, la photographie (sauf les diapositives) et les « images-lumière » qui résultent de la présence de lumière sur un support ayant une source localisable. En insistant sur le faisceau lumineux de la projection au point d'origine bien visible, Claude Simon souligne l'appartenance des images cinématographiques aux images-lumière. En fait, la principale distinction qui est faite dans l'œuvre entre les images cinématographiques et les

${ }^{6}$ Claude Simon, "Attaque et stimuli " (entretien inédit) in Lucien Dallenbach, Claude Simon, Seuil, 1988, p. 172.

${ }^{7}$ Cf. Jacques Aumont, L'image, Nathan, 1990, p. 135. 
autres (peinture et photo en particulier) n'est pas le mouvement. Cette frontière n'en est pas une puisque, dans un trajet d'inversion, les images fixes s'animent et les films se figent. La différenciation est plutôt à opérer grâce à la typologie de Jacques Aumont. Les photographies, cartes postales, tableaux, sont des images opaques faites pour être vues par réflexion et que l'on peut toucher et manipuler. Les films projetés sont des images-lumière, elles sont de passage, ne s'intégrant pas à la surface qui leur sert de support et restant sensibles à chacune de ses modifications. La projection lumineuse apparaît bien fragile et l'image-lumière comme plus immatérielle et inaccessible qu'une autre. L'auteur exprime d'ailleurs cette idée en faisant du cinéma "l'inaccessible rêve scintillant» $(R F, 357)$, les spectateurs ne pouvant cependant pas s'empêcher d'essayer de le saisir, "s'interposant dans le rayon lumineux, projetant leurs ombres immenses et mouvantes sur l'écran ». Les images cinématographiques chez Claude Simon s'inscrivent donc dans la tradition qui voit dans le cinéma le monde des rayons et des ombres (pour reprendre le titre d'un livre de Desnos sur le cinéma) : "Assis dans l'obscurité mystérieuse du cinéma, je regarde se succéder sur le rectangle lumineux, plates, impalpables, éphémères, les images $[\ldots] »(C R, 96)$.

Les images-lumière vont dépasser le cadre purement cinématographique et inspirer d'autres descriptions. Ainsi, dans La Bataille de Pharsale, ce sont les caractéristiques de l'image-lumière qui indiquent le caractère inaccessible de la femme qui est vue "sur d'impalpables ténèbres comme une projection purement lumineuse comme une apparition sans consistance " $(B P, 576)$. Ainsi les images-lumière, fugitives et impalpables, vont contaminer d'autres perceptions présentes dans le récit, devenant bientôt l'outil privilégié de descriptions d'images hallucinatoires, tendant vers le fantastique.

\section{Réversibilité et analyse}

Enfin, il ne faut pas oublier qu'une profonde unité lie ces deux moments filmiques, tournage et projection, dans l'œuvre de Claude Simon. On passe sans arrêt de la projection au tournage et inversement, du tournage à la sonnette annonçant le début de la séance :

Lorsque les rampes des projecteurs se rallument l'une après l'autre, l'appareil de prises de vues a été roulé en avant et dans le viseur s'en- 
cadrent en gros plan la tête, le buste et les bras de la femme [...]. La sonnette annonçant le début imminent de la séance s'est tue depuis quelques instants quand parvient du dehors le tapage de deux voitures qui semblent se poursuivre $[\ldots] .(T, 823)$

Cette alternance crée une dynamique irrépressible dans Triptyque où, de proche en proche, les images cinématographiques se multiplient et finissent par envahir tout le roman. Ainsi, les deux amants de la ville du nord deviennent semblables à des images projetées :

Le visage blafard et renversé de la femme adossée au mur dans l'étroite impasse semble flotter sur l'ombre, faiblement éclairé non par le pinceau lumineux qui jaillit horizontalement du vasistas et passe bien au-dessus de sa tête, mais par la diffraction de sa lumière sur les gouttes de pluie qui continuent de le rayer de stries argentées. $(T, 775)$

En même temps que la projection officielle qui a lieu dans la salle, se déroule une projection secrète. Le faisceau de lumière se dédouble, l'un donnant à voir sur l'écran des images du film «le Commissaire Bastianni » et l'autre (qui sort du vasistas) éclairant la scène d'amour entre la serveuse et le jeune marié. Elle s'approprie alors des caractéristiques cinématographiques : comme une image projetée, le visage de la femme perd de sa concrétude, "semble flotter ». Notons également la pluie qui évoque les rayures de la pellicule (métaphore souvent utilisée par Claude Simon). Réversibilité de la projection qui donne à voir son envers, c'est-à-dire un autre film. Feuilletage d'images réversibles l'une dans l'autre, à l'infini, l'auteur nous proposant un véritable labyrinthe filmique.

D'autre part, cet aller-retour permanent entre tournage et projection va jusqu'à l'interpénétration et démêler ces deux temps devient alors bien difficile :

La surface rectangulaire de l'écran est maintenant divisée en compartiments à peu près carrés, trois dans le sens de la largeur, deux et demi dans le sens de la hauteur. [...]. Deux mains osseuses, à la peau terreuse et criblée de taches de son jaillissent sur la gauche de l'écran. [...]. Enfin elle extrait un quatrième lapin et la caméra se rapproche lentement tandis que la femme se redresse, élevant 
en même temps le lapin qui se détache maintenant au-dessus du clapier, la caméra s'immobilisant alors, cadrant, à gauche, le profil couturé de rides [...]. (T, 841-844)

Nous nous trouvons à la fois au moment de la projection, puisqu'on évoque l'écran et les repères spatiaux qu'il induit (« gauche de l'écran »), mais aussi au moment du tournage avec la description précise des mouvements de la caméra et une spatialisation dépendant cette fois de la caméra (" cadrant à gauche »). Selon Pascal Bonitzer : « Si pour le sens commun, au cinéma l'enregistrement est premier par rapport à la projection parce qu'en effet il l'est chronologiquement, pour le cinéaste c'est la projection qui est première, principale, c'est l'image finale qui infléchit a priori tout le système. $\|^{8}$. Claude Simon réfléchit en spectateur, mais en spectateur qui se veut cinéaste et part presque systématiquement de l'image finale, pour mieux réfléchir aux moyens à employer pour l'obtenir. De fait, on a vu que le présent de la projection portait en creux le présent du tournage. L'auteur donne à voir non seulement l'image cinématographique mais son envers, ses processus de fabrication

Sans doute la caméra a-t-elle été hissée au sommet, soit d'un clocher, soit encore de l'un de ces échafaudages de poutrelles métalliques qui s'élèvent au-dessus du puits d'une mine et qui dominent l'agglomération, mais en tout cas dans l'axe de la longue artère, car l'on découvre celle-ci en vue plongeante, faiblement éclairée de loin en loin par les réverbères. $(T, 819)$

À partir de l'image cinématographique, plutôt que de reconstituer une histoire, le spectateur simonien cherche à découvrir le placement caméra. Il s'agit d'analyser au plus près ces images, de les scruter à la loupe jusqu'à en comprendre l'origine.

Claude Simon semble éprouver un véritable plaisir à décrire la " machine cinéma », privilégiant deux temps dans le processus de fabrication et de vie du film, le tournage et la projection. Le moment du tournage est décrit avec un certain réalisme, Claude Simon évoquant avec justesse son atmosphère. Dans ces descriptions de la

\footnotetext{
${ }^{8}$ Pascal Bonitzer, Peinture et cinéma. Décadrages, Cahiers du cinéma, éd. de l'Étoile, 1995, p. 22 .
} 
façon dont se déroule un tournage, un élément est davantage mis en exergue : le studio. La construction d'un espace factice, qui est là pour s'exhiber, met en relief la question de l'illusion par et pour l'image. En ce qui concerne le moment de la projection, ici encore, Claude Simon fait preuve d'une grande familiarité avec la technique mise en œuvre, l'auteur privilégiant les conditions de projections difficiles, les pannes et les déchirures de pellicule qui permettent de mettre en valeur la matérialité du film. Entre facticité et matérialité, une profonde unité lie ces deux moments filmiques, tournage et projection. On passe sans arrêt de la projection au tournage et inversement, dans une dynamique faite de réversibilité. Au cœur de cette fascination brille la puissance manipulatrice du cinéma. Ainsi la technique du dispositif cinématographique influe sur le rythme et les thématiques du texte simonien, posant en particulier les problèmes des rapports entre fiction, réalité, illusion et truquage. 